\title{
Depression in US Hispanics: Diagnostic and Management Considerations in Family Practice
}

\author{
Roberto Lewis-Fernández, MD, Amar K. Das, MD, PhD, César Alfonso, MD, \\ Myrna M. Weissman, PhD, and Mark Olfson, MD, MPH
}

US Hispanics, currently the largest minority group in the country, face disparities in the recognition and treatment of major depression. This article provides an evidence-based review of the prevalence, presentation, and management of major depression in primary care in this heterogeneous ethnic group. Under-recognition of depression in adult Hispanic Americans may be related to language differences, health literacy barriers, somatic presentations, and use of cultural idioms of distress. Hispanic patients are often agreeable to treatment but as a group may tend to prefer psychotherapy or combined counseling and medication to pharmacotherapy alone. Recent studies have found both psychological and pharmacologic treatment options are efficacious in this minority group. Treatment adherence should be actively monitored to help ensure its effectiveness. To help reduce the number of depressed Hispanic patients who pass through primary care undetected or inadequately treated, family physicians should make a concerted effort to increase their awareness of the presentation of major depression in this population and actively promote adherence to effective treatments. ( $\mathrm{J}$ Am Board Fam Pract 2005;18: 282-96.)

US Hispanics, now the single largest minority group in the country, ${ }^{1}$ face significant disparities in health and health care. ${ }^{2}$ In the treatment of mental disorders, there is a growing awareness of inadequate care for major depression among US Hispanics. ${ }^{3,4}$ Family physicians and other primary care providers can play a critical role in addressing such racial and ethnic health disparities. ${ }^{5}$ Hispanics, or Latinos, are twice as likely to seek help for mental health problems from primary care providers as from mental health specialists. ${ }^{6}$ Their use of these specialists is significantly lower than that of majority whites, even after adjusting for differences in sociodemographic characteristics and rates of psy-

Submitted, revised, 1 March 2005.

From the Department of Psychiatry, Columbia University, New York (RL-F, CA, MMW, MO), the New York State Psychiatric Institute, New York (RL-F, CA, MMW, MO), and Department of Psychiatry Stanford University School of Medicine, Stanford, California (AKD).

Funding: This project was supported by an investigatorinitiated grant from Eli Lilly \& Company, Indianapolis (Dr. Olfson) and the Columbia Center for the Health of Urban Minorities (NCMHHD MD000206-01).

Corresponding Author: Roberto Lewis-Fernández, MD, New York State Psychiatric Institute, 1051 Riverside Drive, Rm 3200 (Unit 69), New York, NY 10032 (e-mail: rlewis@ nyspi.cpmc.columbia.edu). chiatric disorder. ${ }^{7-9}$ In contrast, Hispanics use the general health sector for mental health problems at a rate that is similar to whites once differences in level of psychiatric need are taken into account. ${ }^{7,9,10}$ Previous studies ${ }^{11-13}$ indicate that major depression is a common and disabling disorder among Latino patients in primary care. A synthesis and review of findings is provided concerning the prevalence, diagnosis, and management of depression in adult US Hispanics.

US Hispanics are heterogeneous, and differences among subgroups are important to recognize in assessing mental health needs. Most Latinos have a common ancestry in people speaking the Spanish language or emigrating from Latin America. By origin, Mexican Americans form the largest subgroup (almost 6 in 10 Latinos) with Puerto Ricans as second largest (approximately 1 in 10) and Cuban Americans the third largest (approximately 1 in 30) in the United States. ${ }^{1}$ Recent immigration, family-oriented cultural values, and poverty are common and shared features among the various subgroups. However, there is a wide range of socioeconomic characteristics in the Hispanic community. Because of marked undercount of migrant Hispanics on the decennial census, con- 
troversy remains as to the precise proportion of US Hispanics who are foreign-born. Estimates range from $40 \%$ to $60 \%$ of the Latino population. ${ }^{14}$ Once the high proportion of US-born Hispanics with at least one immigrant parent-estimated at $20 \%$-is also taken into account, the salience for this community of recent immigration experience and traditional cultural values comes into focus. "Circular" migration patterns, characterized by frequent back-and-forth travel to Latin America, ${ }^{15}$ also maintain US Latinos' awareness of their cultures of origin.

\section{Prevalence}

Major depression is the mental health problem most frequently diagnosed by primary care physicians. ${ }^{16} \mathrm{~A}$ number of national, community, and primary care studies have examined the rate and clinical risk factors of major depression in US Hispanics.

\section{National and Community Studies}

The research literature on the epidemiology of depressive symptoms and depressive disorders among Hispanics in the United States has grown significantly over the past 2 decades. Early community studies indicated that Hispanic populations have higher rates of depressive symptoms than majority white populations. ${ }^{17}$ The results of the Epidemiologic Catchment Area study (ECA) from the Los Angeles site ${ }^{18}$ (which oversampled Mexican Americans), and of the National Comorbidity Study $(\mathrm{NCS})^{19}$ indicated, however, that overall rates of major depression and other psychiatric disorders were similar between US non-Hispanic and Hispanic populations. The recent replication study of the NCS found that Latinos were at a nonsignificantly increased risk of major depression in comparison with non-Latino whites. ${ }^{20}$ In contrast, the rate of major depression was significantly lower in the African American population than in either the Latino or white population. An important limitation of the NCS and NCS-R is that they were conducted only in English and so did not include less acculturated Latinos.

The risk of major depression seems to be greater for US-born Mexican Americans than for Mexican immigrants. An early epidemiologic study found that Mexican immigrants have a significantly lower rate of major depression than US-born Mexican
Americans. ${ }^{21}$ In this study, which was conducted in Spanish and English, there was a nonsignificant trend toward increased risk of major depression in the more acculturated groups. Possible explanations include that Mexican American immigrants tend to experience a lower sense of deprivation than US-born Mexican Americans, immigrants retain a stronger family orientation or other cultural values than US-born persons that tend to protect them from depression, or that immigration tends to select for mental health. Some research, however, casts doubt on this selection hypothesis. In this study, which also included English- and Spanishspeaking Latinos, Mexican immigrants had approximately one half the rate of depression of US-born Mexican Americans. ${ }^{22}$ It is interesting that the rate of depression among Mexican immigrants resembled the rate in Mexico City, and the rate of depression of US-born Mexican Americans resembled the overall rate of depression in the United States. ${ }^{22}$ Recent community epidemiologic studies further indicate that the 12-month prevalence of mood disorders is significantly higher in the United States than in Mexico, Colombia, or Spain. ${ }^{23}$

A relationship between nativity and risk of depression, best established among Mexican Americans, may also extend to other Hispanic groups and to psychiatric disorders in general. In a study of Cuban Americans, the lifetime rate of US-born Cuban Americans (5.1\%) was significantly higher than for Cuban Americans born in Cuba. ${ }^{24} \mathrm{An}$ analysis of the National Comorbidity Study also revealed that even after controlling for age, income, and education, US-born Hispanics had a significantly higher risk of psychiatric disorder than foreign-born Hispanics. ${ }^{25}$ A significant association was observed among separate analyses of Puerto Ricans, Mexican Americans, and other Hispanics. ${ }^{25}$

A comparative study of Puerto Ricans living in poor residential areas in Puerto Rico and New York City revealed similar levels of clinically significant self-report depressive symptoms in each group. ${ }^{26}$ High levels of depressive symptoms were common among Puerto Ricans living in Puerto Rico $(29.1 \%)$ and Puerto Ricans living in New York City $(28.6 \%){ }^{26}$ This finding cautions against over-generalizing the hypothesis that acculturation increases the risk of depression and suggests a need to examine in greater detail cultural differences between and within Hispanic subgroups and eval- 
uate cultural variation across self-report and structured research interviews of depression.

The Hispanic Health and Nutrition Examination Survey (Hispanic HANES), which was conducted between 1982 and 1984, was the first population-based health survey of US Hispanics of Mexican, Puerto Rican, and Cuban origin conducted in the 3 main geographical regions of the country with large Hispanic populations. ${ }^{27}$ The Hispanic HANES revealed that among respondents 20 to 74 years of age, the 1-year prevalence of major depression was significantly higher in Puerto Ricans (6.9\%) than in Cuban Americans (2.5\%) or Mexican Americans (2.8\%). ${ }^{28}$ The underlying basis of these group differences remains poorly understood. Results from the recently completed National Latino and Asian American Survey, a community-based household study with nationally representative samples of US Latinos and Asian Americans, are eagerly awaited to help clarify the extent of intra-Hispanic variability in depression prevalence and in the moderating effect of acculturation.

\section{Primary Care Studies}

Over the past 2 decades, a number of studies have examined major depression and other mental health problems among Latino primary care patients. In an early study of depression among Latinos, researchers approached Hispanic patients at a general medicine clinic at San Francisco General Hospital. ${ }^{31}$ Most (72\%) had emigrated from Central America, whereas fewer (16\%) were immigrants from Mexico. Twenty-eight percent of patients were diagnosed with major depression.

A second study assessed depression and anxiety with structured diagnostic interviews in a family health center in San Antonio, Texas, where approximately 3 of 4 patients were Mexican Americans. ${ }^{11}$ The rate of depression was more than 3 times greater in white women (32\%) than in Mexican American women (10\%). Among all women, 4 of 5 of whom were of Hispanic origin, depression was associated with multiple somatic symptoms of unknown medical cause. There were no significant differences between white, African American, or Mexican American men. Because respondents' place of birth was not reported, it is unclear whether the lower rate of depression in Latino women in this study was associated with foreign birth or with other risk or selection factors. Hoppe et $\mathrm{al}^{11}$ also conducted similar diagnostic interviews in 5 family medicine clinics in Monterrey, Mexico. Age-adjusted rates of depression among Mexican American women in San Antonio (8.3\%) were twice that of women living in Monterrey (3.8\%). This is consistent with the hypothesis that acculturation is a risk factor for depression in Mexican Americans coming to primary care clinics.

Escobar et $\mathrm{al}^{32}$ determined rates of mental disorders assessed with a structured diagnostic interview and examined the effects of acculturation in a study of somatization among primary care patients at a university-based clinic in Anaheim, California. The sample consisted of 1456 patients. Almost 2 of 3 patients were of Hispanic ethnicity, either immigrants from Mexico or Central America or USborn Mexican Americans. There were lower rates of depression as well as better overall physical functioning in Hispanic immigrants than in US-born Latinos. ${ }^{33}$

In another large primary care study at a university-affiliated clinic in New York City, ${ }^{13} 1007$ patients were systematically recruited to participate in a mental health screening with a self-report instrument. Nearly three quarters of this sample consisted of patients who were US Hispanics; more than $90 \%$ of these were born in Latin America, largely in the Dominican Republic. Using the PRIME-MD Patient Health Questionnaire, the study found high current rates of depression among Latinos (22\%) in relation to non-Hispanic whites (12\%) and African Americans (10\%). The difference between Latinos and whites was not statistically significant, which may be related to the small percentage of whites in the sample. The elevated rate of depression among immigrant Hispanics in this study, relative to whites and African Americans, brings up a similar caution as for nonprimary care studies against over-generalizing the possible pathogenic effect of acculturation. More research with diverse Latino subgroups comparing the results of self-report and diagnostic instruments is needed to clarify this issue.

\section{Clinical Correlation: Assessment of Acculturation in Hispanic Immigrants and Their Offspring}

The strength of recommendation in favor of assessing the acculturation level of Hispanic patients is $\mathrm{C}$ (inconsistent results of good-quality descriptive studies). 
- Establish the patient's language preference for the interviews. Patients with limited English capacity may overstate their fluency because of its social desirability.

- Inquire directly about place of birth, age of migration to US if foreign-born, and degree of back-and-forth travel.

- Remember that contemporary theories of acculturation reject a "zero-sum" model, which mistakenly assumes that as people become more fluent in the new culture, they necessarily become disconnected from their culture of origin. ${ }^{29}$ Instead, multiple cultural attachments are possible, especially over time, and can impact illness interpretation and treatment negotiation.

- Assess the degree of encapsulation in Latino enclaves, because this may retard exposure to new cultural experiences and ethnic groups despite longstanding US residence.

- Assess the ethnic composition of the patient's neighborhood, because immigrant Hispanics acculturate to a patchwork of local cultural contexts rather than to a homogenous Anglo society.

- Find out about patients' premigration experience to help contextualize their clinical expectations (eg, urban versus rural, occupational background, exposure to mass media, past use of biomedical versus folk health systems).

- Investigate the circumstances of migration, such as the presence of relatives already here, political circumstances surrounding departure and reception in the United States, and relative relevance of push-pull factors.

- Keep in mind that parts of the country (eg, the Southwest) were colonized by Latinos before the arrival of the US flag. For new migrants to these settings, acculturation involves adjusting to longstanding Mexican American cultures as well as other non-Latino groups.

- Assess patients' definitions of who may be considered part of the "family" and how involved they should be in health care issues. In general, culturally traditional Latinos have more inclusive notions of family composition than more acculturated Hispanics.

- Evaluate the role of intergenerational and gender-related conflicts related to acculturation in precipitating depressive symptoms. These conflicts typically involve changing views across generations or between spouses about expected levels of family reciprocity and support, illness inter- pretations, and views regarding sexuality, spirituality, or gender roles. It is useful to ask patients about their children's experience of acculturation to establish the salience of intergenerational conflicts.

- Assess the impact of acculturation on diet. Partly because of inaccessibility of traditional ingredients and demanding work schedules, many Latino immigrants abandon healthier traditional diets in favor of prepared foods with higher fat, salt, and sugar content. ${ }^{30}$

\section{Clinical Vignette: Depression in Primary Care}

Esperanza, a 54-year-old woman who migrated from Mexico to the United States 20 years ago, presents to a family practice clinic with type 2 diabetes mellitus and hypertension. She also describes "suffering from nervios (nerves)" and fear of becoming "weak" (débil). Although she implies a contrast with her lifelong self-perception as "a strong person"-able to withstand stressors with little support, including crossing the border illegally and raising her children with limited finances-this complaint is initially taken to refer only to reduced physical strength and stamina. Not mentioned at first is her sadness and frustration at not having achieved the economic security she sought by migrating and about ongoing conflicts with her husband and one of her sons over their substance abuse. Her presenting complaint instead is about feeling sofocada (out of breath), which she describes as a mixture of shortness of breath, chest pressure, heat in the chest and face, and weakness/fatigue. She describes feeling tense and nervous (nerviosa) but initially does not report her irritability, which contradicts her wish to see herself as una persona tranquila (a tranquil/equanimous person). Her complaints are discussed mostly in somatic terms, with prominent palpitations, dizziness, and headache in addition to the initial symptom of being sofocada.

The somatic focus of the presenting complaints leads initially to a negative work-up centered on potential cardiac and respiratory conditions. Further evaluation reveals a strong depressive component, including anhedonia, low energy, difficulty taking care of her children and cooking for the family, decreased sleep, poor concentration, elevated appetite, and weight gain. However, attempts to address her major depressive disorder with antidepressants and a psychiatric referral meet with 
considerable resistance. She is concerned about using "strong" psychotropic medications, such as antidepressants, because of their perceived addictiveness and harmfulness: curan por un lado pero dañan por el otro (they cure on the one hand but cause harm on the other). Instead she prefers a sleeping pill, but the clinician is concerned about its limited efficacy and addictive potential when used as depression monotherapy. Esperanza is also opposed to a psychiatric referral because of its stigma: "I am not crazy (loca)." She also worries the psychiatrist will disapprove of her coping system, which includes Evangelical services and curandero (folk healer) treatment. She is more open to receiving counseling, but time and financial limitations are substantial barriers. Likewise, diet and exercise recommendations are initially not taken up because, as the household cook, she is reluctant to impose the new diet on the whole family. Her depressive symptoms and the lack of safety in her neighborhood make exercise difficult.

The family physician was much more successful when (s)he pursued a new approach that actively engaged the patient's illness interpretations and expectations. Esperanza was more willing to implement antidepressant therapy and dietary modification after an intervention that:

1. focused the evaluation on her nervios illness, framed as an "alteration" of the nervous system because of her prolonged suffering, including her frustration over her migration experience and her family conflicts. Interpreting the depressive symptoms as signs of nervios placed the burden of causation on the overwhelming character of the precipitating stressors, rather than on an internal diathesis;

2. validated her view of somatic symptoms as the result of nervios, which also worsens the impact that the diabetes and hypertension have on her functioning;

3. reinterpreted antidepressants as nonaddictive "medications for the nervous system," which can be supplemented with intermittent use of sleeping medication. In contrast to her view, the sleeping pill was presented as being more addictive than the antidepressant, and thus to be used only sporadically;

4. reframed the negative physical work-up as evidence that the nervios illness has not yet caused permanent damage to other organ systems. She remains a "strong" person, who is suffering from a physical condition because she has endured so many stressors;

5. expressed willingness to learn more about other forms of health knowledge, including spiritual healing systems that seem exclusive to biomedicine (and to each other) only when understood superficially and that may act as very useful supplements to the clinician's approach;

6. organized a family level intervention with the help of a dietician and Esperanza's adult daughters that stresses the value of specific aspects of traditional Mexican diet that may be both acceptable to the family and heart-healthy (eg, complex carbohydrates, fresh produce, meat as noncentral part of meal).

\section{Diagnostic Evaluation}

Major depression is diagnosed clinically by the presence of characteristic somatic symptoms in the setting of either depressed mood or diminished interest in pleasurable activities during a 2-week period. Because primary care providers face time constraints in making a full assessment, some choose to use brief screening instruments for depression in the primary care setting, such as the PRIME-MD, available in English and Spanish. ${ }^{34,35}$ In addition to being affected by institutional barriers relevant to all ethnic groups, like financial disincentives to specialty referral and stringent clinician productivity quotas, detecting major depression in low-income Latinos may be complicated by language differences, health literacy barriers, somatic presentations, and use of cultural idioms of distress. A major cause of misdiagnosis and inadequate treatment of depression in this population is the mismatch between the professional culture of the medical system and the folk culture of many US Latinos regarding what to expect of the presentation, evaluation, and treatment of depressive symptoms.

\section{Language and Health Literacy Barriers}

Nearly half of Latinos who speak primarily Spanish in their homes report problems speaking English. ${ }^{36}$ Several studies have found that limited English proficiency is a significant barrier to health care access and quality care. In a study of hypertension and diabetes, ${ }^{37}$ monolingual Latino patients whose 
physicians spoke Spanish reported better well-being and functioning than those patients whose physicians were not fluent in Spanish. A national study of racial and ethnic disparities in access to health care $^{2}$ found that Spanish-speaking Hispanics were significantly less likely than majority whites to make a physician or mental health visit. In contrast, the pattern of health care access for English-speaking Hispanics was not significantly different from that of non-Hispanic whites. In a West Coast survey of 7093 outpatients receiving medical care from a physician group association, Spanish-speaking Latinos reported significantly greater dissatisfaction with the communication skills of their medical providers than English-speaking Latinos and nonHispanic whites. ${ }^{38}$

Limited English proficiency among US Latinos may also indicate more fundamental differences in the interpretation and use of health-related information, possibly because of lower formal education and differing cultural understandings of illness. In studies in 2 large, urban public hospitals, $62 \%$ of Spanish-speaking versus 35\% of English-speaking patients were found to lack the health literacy skills needed to function in the health care setting in a manner consonant with the expectations of evidence-based clinicians. ${ }^{39}$ Health literacy may be defined as the degree to which a person is able to obtain, process, and understand the basic health information needed to make appropriate health care decisions in a modern biomedical setting. ${ }^{40}$ Low health literacy can compromise treatment efforts by hindering patient-clinician communication. ${ }^{39,41}$ In a study of 408 English- and Spanishspeaking patients with diabetes mellitus in a San Francisco primary care clinic, Spanish-speakers were significantly over-represented in the group with inadequate health literacy. Patients with inadequate health literacy on a standardized instrument were more likely than patients with adequate health literacy to have higher hemoglobin $\mathrm{A}_{1 \mathrm{c}}$ levels and to develop retinopathy, after adjusting for sociodemographic characteristics, social support, and several clinical factors. ${ }^{41}$

Securing staff to provide Spanish interpretation may be one of the greatest challenges in combating disparities in mental health care access. ${ }^{42,43}$ Many staff members in primary care offices are not trained to provide culturally competent services. In one study, ${ }^{44}$ family members were the most common means of overcoming language differences between patient and physician. Reliance on family members as interpreters may lead to serious problems in confidentiality in evaluating issues such as suicidal ideation and sexual symptoms. Physicians who use trained interpreters report higher quality of patient-physician communication than those who use other means. ${ }^{44}$ In one large HMO in the Northeast, Spanish-speaking patients who received a professional interpreter service during clinical care made more office visits, filled more prescriptions, and accepted more preventive services than those who did not have such a service. ${ }^{45}$

\section{Clinical and Policy Considerations}

Growing legal and professional requirements about the evaluation and appropriate management of patients' health literacy and language capacity are increasingly affecting all aspects of health care. ${ }^{46}$ According to the Department of Justice, it might constitute national-origin discrimination for a hospital to only make available English versions of forms or documents and be unable to help those "who don't speak, write, or understand English well enough." ${ }^{47}$ JCAHO also requires hospitals to establish a mechanism ensuring that information can be understood by patients. ${ }^{48}$ Recommendations to assess and manage low health literacy and how to access and work with interpreters include the following.

\section{Health Literacy}

The strength of recommendation in favor of assessing health literacy is B (consistent results of goodquality descriptive studies).

- Assess patients' level of health literacy during the initial interview by eliciting years of schooling and possible difficulties filling out office forms. Patients with low health literacy may be reluctant to volunteer their limited understanding; asking patients who seem unclear about verbal instructions to paraphrase what was said is a nonstigmatizing way of overcoming this resistance. Certain scales may also help measure illness-specific health literacy, such as the Test of Functional Health Literacy in Adults, adapted for hypertension and diabetes. ${ }^{39}$

- Evaluate reading capacity before giving written material. To facilitate comprehension, it is best to keep all written text at a 6th grade level or below. Diagrams and Spanish-language videos 
may be helpful adjuncts for patients with low health literacy or poor English proficiency. ${ }^{48}$ Some patients may use polite evasions to avoid embarrassment, such as claiming they forgot their glasses to cover up their inability to read.

- Demonstrate any procedure prescribed. Ask patients to perform the procedure in front of you.

- Have bilingual health materials available.

- If possible, have staff members assist with filling out forms.

- If the patient agrees, include family members in the discussion about treatments and procedures, so that they can assist the patient in carrying them out.

\section{Interpreter Services}

The strength of recommendation in favor of using trained interpreters is A (consistent results of goodquality patient-oriented studies).

- Avoid using family, friends, children, nonclinical staff, and persons from the waiting area.

- Use trained medical interpreters instead of untrained Spanish-speaking staff.

Trained interpreters:

- learn to translate word-for-word rather than by summarizing and paraphrasing, which can lead to misunderstanding

- are aware of intra-Latino vocabulary differences

- take account of patient education level in translating clinicians' comments. Referred to as establishing the appropriate "register," this ensures understandability of the translation ${ }^{49}$

- do not insert own material or interpretations without informing others, but rather let both interlocutors know about suggestions for clarification if they detect an obvious misunderstanding

Resources for interpreter services:

- Local/state departments of health

- Many teaching hospitals

- National Council on Interpreting in Health Care (http://www.ncihc.org)

- Society of Medical Interpreters (http://www. sominet.org)

- American Translators Association (http://www. atanet.org)
- Austin Area Translators and Interpreters Association (http://www.aatia.org)

- Massachusetts Medical Interpreters Association (http://www.mmia.org)

- California Health care Interpreters Association (http://www.chia.ws)

- Community and Court Interpreters of Ohio Valley (http://www.ccio.org)

- Nebraska Association for Translators and Interpreters (http://www.natihq.org)

- Telephone interpreter lines (search "telephone interpreter" on the web for various suggestions).

- Community volunteers may be willing to receive training in interpretation skills.

\section{Somatic Presentation}

The presentation of primarily somatic symptoms to describe mental health distress can pose a problem in the detection of depression in primary care. Somatic presentations of depression are frequent across different cultural and ethnic groups. ${ }^{50,51}$ This presentation may be particularly common among Hispanic subgroups, who have been found to present psychological distress in the form of physical symptoms. ${ }^{52-56}$ In one study, Mexican American women were found with a structured diagnostic interview to be more likely to report somatic symptoms of depression than white women. ${ }^{57}$ Another study found that Puerto Ricans had higher rates of somatization compared with Mexican Americans and non-Latinos. ${ }^{58}$ Data from the Hispanic HANES revealed that the constellation of depressive symptoms among Hispanics who are primarily Spanish-speaking tend to involve less differentiation of mood and somatic symptoms than their Hispanic counterparts who are primarily English-speaking. ${ }^{59}$ A somatic presentation style may be particularly common in general health settings, partly because of patients' expectation that clinicians will focus on the physical components of illness.

Current diagnostic criteria for major depression incorporate both psychological and somatic symptoms. However, some patients who have major depression with somatic presentations may express little sadness or depressed mood, especially during initial assessments. In the general medical setting, some physicians may have difficulty detecting predominantly somatic presentations of depression, resulting in unnecessary diagnostic and laboratory 
procedures. Evaluating somatic symptoms with an approach that considers biological, psychological, and social factors can help primary care physicians to detect cases of depression that have predominantly somatic presentations. ${ }^{60}$

\section{Clinical Correlation: How to Assess Somatic Presentations in Latinos}

Many Latino somatizers welcome a clinical interpretation that transcends mind-body dualism and links somatic symptoms, emotional reactions, and real-life stressors. The following are recommendations to assess Latino patients suffering from depression and unexplained medical symptoms. The strength of recommendation in favor of this assessment is $\mathrm{C}$ (expert opinion, case series).

- Inquire about the patient's understanding of the somatic symptoms: are they simply more visible signs of acknowledged emotional distress; "the real problem" in the absence of any perceived psychiatric difficulties; the cause of secondary emotional problems; or a way of asking for help, an entry point for discussion of partially understood social or interpersonal conflicts?

- Clarify patients' use of specific cultural idioms of distress to describe the somatization process (eg, nervios [nerves], anger-related illnesses such as bilis [bile] or cólera [rage], conditions associated with the hot-cold theory of disease such as pasmo, or afflictions attributed to the abnormal circulation of air in the body, such as mal aire [bad air] or gases). ${ }^{61,62}$

- Some patients may emphasize somatic symptoms to negotiate a biomedical system that tends to separate somatic from emotional problems and attributes greater ontological value to the physical. These patients may actually be trying to "speak the language" of the biomedical practitioner, especially given the relative unavailability of psychosocial treatments, such as psychotherapy, to low-income patients.

- Recognize that somatic symptoms are real and not imagined.

- Explore physical symptoms in the context of stressors with open-ended questions such as: "What are the problems that you are facing now that create difficulty or distress?"

- Rule out alternative physical disorders in the medical differential diagnosis, but if depression or other psychiatric disorders are evident and physical symptoms are clearly medically unexplained, avoid unnecessary referrals or lengthy work-ups. Discussion of negative laboratory or imaging tests with the patient is usually helpful, particularly if presented as part of a preplanned evaluation to rule out nonpsychiatric causes. Not conducting any tests, however, may be taken as a sign of negligence or lack of caring.

- Discuss with the patient your understanding of their physical distress in relationship to their life situation and stressors. Many patients will find a biopsychosocial interpretation helpful and respectful, even if they disagree with the relative weighting the clinician ascribes to the different components.

- Recognize that some patients with somatization are unable to filter out their perception of irrelevant bodily stimuli and their awareness of bodily sensations may get reported as physical distress. This process, called somatosensory amplification, interferes with the patient's ability to focus on the external environment because of a hyperawareness of internal sensations.

- Consider that a minority of somatization patients may also suffer from alexithymia. Alexithymia is an extreme inability to verbalize feelings or emotional states. These patients may displace emotions and express them purely or primarily with physical symptoms.

- Patients with somatization feel relief when they perceive that their physicians believe and listen to them. Appearing incredulous or dismissive only escalates their distress. Listening attentively, with curiosity, patiently, and serenely often has a therapeutic effect.

\section{Depression-Related Cultural Idioms of Distress}

In addition to a the frequent experience of somatization, many depressed Hispanics present to primary care and specialty mental health complaining of other culturally patterned idioms of distress. ${ }^{62,63}$ These are linguistic and bodily styles of expressing and experiencing illness, and affliction more generally, that do not have a one-to-one relationship with diagnostic categories. ${ }^{64}$ Many low-income Hispanics, for example, describe a constellation of depressive, anxiety, somatic, and dissociative symptoms known as nervios (nerves) illness. With some gender, age, and Latino subgroup variation, reports 
of nervios typically signal particular patient and family expectations about causation (eg, "alteration" of the anatomic nerves due to interpersonal suffering), symptoms (eg, trembling, fatigue, malaise, "brain-ache" (dolor de cerebro), perceptual distortions such as hearing noises or seeing shadows (known as celajes), treatment (eg, as-needed medications, "advice," advocacy with government programs), and outcome (eg, fear of recurrence, longstanding vulnerability to minor stressors). ${ }^{62}$ Knowledge of these cultural idioms can facilitate rapport and psychoeducation, help justify and guide the diagnostic work-up and minimize the risk of misdiagnosis. For example, clinicians aware that some depressed Latinos may express acute fits of emotionality known as ataques de nervios (attacks of nerves) in response to interpersonal stressors can more easily prevent their misdiagnosis as syncopal, ictal, or panic episodes. ${ }^{6,65}$ Likewise, information about the tendency of Latino patients with depression and post-traumatic stress disorder to report nonpsychotic perceptual distortions can reduce the risk of inappropriate antipsychotic medication use in this population. ${ }^{66}$ Eliciting specifically cultural information from patients and their families is a useful part of the clinical evaluation.

\section{Clinical Correlation: The Cultural Formulation Model}

The Cultural Formulation (CF) is a systematic model for cultural assessment that can be performed as part of any clinical encounter. ${ }^{67,68} \mathrm{An}$ outline of its components is presented below and described further in Appendix I of DSM-IV, the diagnostic manual of the American Psychiatric Association. ${ }^{69}$ The CF model is designed to supplement a standard clinical evaluation by highlighting the effect of culture on the patient's identity, personality development, symptomatology, explanatory models of illness, help-seeking preferences, stressors and supports, therapeutic relationships, and outcome expectations. The strength of recommendation in favor of using the CF model is $\mathrm{C}$ (expert opinion, case series). The CF is composed of 5 sections:

\section{Cultural Identity}

Includes 5 subsections: (1) cultural reference group(s), (2) language, (3) cultural factors in development, (4) involvement with culture of origin, and (5) involvement with host culture. This section identifies the particular sociocultural influences on an individual patient, including demographic and socioeconomic subgroup characteristics, as well as the person's own sense of ethnocultural identity. The last 2 subsections compare patients' involvement with their culture of origin to their involvement with the host culture, an essential comparison for migrants and their offspring.

\section{Cultural Explanations of the Illness}

Includes 4 subsections: (1) predominant idioms of distress and local illness categories, (2) meaning and severity of symptoms in relation to cultural norms, (3) perceived causes and explanatory models, and (4) help-seeking experiences and plans. This section examines the cultural factors that affect the experience and interpretation of illness, as understood by the patient, the family, and the social network, including assessing the role of traditional nosologies (eg, idioms of distress, causes) and local evaluations of severity. This section also explores patients' rationales for past help-seeking choices and current treatment expectations and concerns, including about utilization of general health and specialty services.

\section{Cultural Levels Related to the Psychosocial Environment and Levels of Functioning}

Includes 3 subsections: (1) social stressors, (2) social supports, and (3) levels of functioning and disability. This section documents the effect of culture on the stressors to which patients are exposed, their reactions, their social supports, and the contexts against which their functioning should be measured.

\section{Cultural Elements of the Clinician-Patient Relationship:}

This section self-reflexively examines the effect of the individual and professional characteristics of the clinician, including his/her ethnoracial attitudes, on the patient's symptom presentations, illness attributions, health care utilization, and outcome. The impact of structural and organizational factors, such as of the fragmentation of physical and mental health services, are also assessed.

\section{Overall Cultural Assessment}

The final section of the CF model summarizes the information in the previous sections, focusing on its contribution to diagnosis, treatment, and illness outcome. 
At present, the CF model is used mostly in psychiatric assessments, but work is under way to adapt it to general health settings. Family physicians and other medical clinicians may find the general approach and individual items of the Cultural Formulation useful when assessing cultural aspects of clinical presentations.

\section{Treatment Considerations}

After major depression is diagnosed, the next steps are determining an effective management strategy and ensuring adherence to treatment. For Latino patients, a number of factors need to be considered in determining treatment options.

\section{Counseling and Psychotherapy}

Counseling and psychotherapy are important treatment options in the care of mild to moderate depression. In one study of English- and Spanishspeaking primary care patients, non-Hispanic whites and Latinos did not differ in the percentage choosing treatment with medication or counseling over no treatment. ${ }^{70}$ A more recent study found that Latinos were more likely than whites to want counseling and were less likely than whites to want antidepressant medication. ${ }^{71}$ Research with lowincome Hispanic primary care patients using conjoint analysis, a novel technique grounded in economic theory, revealed a preference for combination treatment (counseling plus medication) over either approach alone. ${ }^{72}$ Respondents also preferred individual to group treatment but did not show a significant preference for treatment setting (general health versus specialty mental health). Whether these stated preferences translate into actual behavioral choices remains unknown. It is also not known whether any particular form of psychotherapy (such as cognitive behavioral or psychodynamic therapy) is more suitable in Latinos than in other ethnic groups. Miranda et $\mathrm{al}^{73}$ have shown that cognitive behavioral therapy (CBT) is effective in reducing symptoms for depressed low-income Latino patients in primary care, although adherence is often poor with longer-term psychotherapy treatment. Depressed adolescents in Puerto Rico showed a higher response rate with interpersonal psychotherapy than CBT, but these results are pending replication. ${ }^{74}$

\section{Medications}

There is a growing awareness that psychotropic medications may have different efficacies and safety/tolerability implications across ethnic and racial groups. ${ }^{75}$ In one study of tricyclic antidepressants, Latinos were found to have more side effects than other groups. ${ }^{76}$ Interethnic differences in drug metabolism by the cytochrome enzyme system may potentially explain such variations in treatment responses, but genetic differences in enzyme activity also exist among persons within an ethnic group. ${ }^{77}$ Dietary and other environmental factors can modify antidepressant metabolism as well, and these factors in Latino immigrants may change significantly through acculturation. ${ }^{77}$ The evidence to date argues for lower ethnic-specific variability in efficacy and safety/tolerability for newer-generation antidepressants than for older tricyclic agents. ${ }^{78,79}$ However, large placebo-controlled ethnic-specific studies are needed to clarify this issue.

Relatively little remains known about the use and effectiveness of antidepressant therapy in US Hispanics. Some studies indicate that Latinos and whites are equally likely to receive antidepressant medication by primary care providers. ${ }^{80,81}$ However, several other studies reveal that primary care physicians as well as psychiatrists are less likely to detect or provide antidepressant treatment for depression in Hispanics than whites. ${ }^{4,73,82,83}$ In one primary care study, for example, a quality-of-care intervention that emphasized antidepressant medications improved the treatment of depression comparably for Hispanics and whites, with the result that the ethnic disparity in treatment at baseline persisted through the intervention despite an overall improvement in the quality of care. ${ }^{84}$ At present, the weight of evidence indicates ethnic-specific undertreatment of depression in Hispanics in primary care and specialty mental health settings. ${ }^{85,86}$

One small open-label study of antidepressants (fluoxetine or paroxetine) in depressed women found that Hispanics and non-Hispanics had similar rates of improvement. ${ }^{87}$ A larger open trial of nefazodone for depression in Spanish-monolingual Hispanics found a response rate for nearly 2 of 3 patients, comparable with historical studies on this antidepressant in predominantly non-Hispanic samples. ${ }^{78}$ Pooled data from 7 randomized, placebo-controlled trials of duloxetine for major depression showed no significant differences in response 
across Hispanic, African American, and white cohorts. ${ }^{79}$ A second randomized controlled trial of antidepressants for depressed HIV-positive patients found that Hispanics were more likely than either African Americans or whites to respond to placebo. ${ }^{88}$ This confirms an early study by Escobar et $\mathrm{al}^{75}$ that also found higher placebo response among Colombians compared with US Anglos.

\section{Clinical Correlation: How to Detect a Likely Placebo Response}

Although the scientific basis for differentiating antidepressant from placebo responses remains understudied, the following may help clinicians distinguish between the 2 :

- Placebo responses tend to be fleeting and inconsistent, whereas antidepressant responses are usually enduring.

- Antidepressants, as a rule, need to be taken for 1 to 3 weeks before the onset of symptomatic relief. A more immediate drug response (within the first week) usually indicates a placebo response.

- Symptomatic relief may result from a medication side effect and not from a placebo or a true antidepressant response. For example, during the first week of treatment, insomnia may be relieved by a drug's sedative properties, in the absence of improvement in other symptoms such as depressed mood.

- Placebo response is more likely in depression of mild to moderate severity than in more severe episodes.

- Patients who present with a first episode of major depression are more likely to develop a placebo response than patients with a history of chronic depression.

\section{Adherence to Treatment}

Even after Hispanic patients overcome entry-related barriers and initiate depression treatment, one of the significant challenges in providing effective care for US Hispanics is to minimize premature treatment discontinuation. In relation to nonLatino whites, Hispanics may have higher rates of early treatment nonadherence for depression. ${ }^{78,88-90}$ In one study, low-income Latinos faced difficulty in making clinic visits because of family burdens, multiple jobs, and transportation problems. ${ }^{78}$ Other important factors that may con- tribute to poor adherence include cultural concerns around stigma of mental health services, ${ }^{91}$ fears of the effects of medications, ${ }^{71}$ and insurance problems. ${ }^{92,93}$ Hispanics have the lowest rate of public or private health insurance in the United States and are more than twice as likely to be uninsured as majority whites $(28.7 \%$ versus $11.9 \%) .{ }^{94}$ Poorer physician-patient communication may also underlie higher antidepressant nonadherence among Latinos. In one study conducted in general medicine and family practice clinics, Hispanic patients were less likely than whites to receive antidepressant information by their physicians and to state information about their medications, resulting in lower short-term medication adherence by Hispanics compared with whites. ${ }^{90}$ The addition of case management to a cognitive behavioral therapy intervention has been shown to reduce dropout rates for all patients and to improved outcomes in Latinos whose primary language was Spanish. ${ }^{73}$ In general, successful adherence- enhancing interventions stress the importance of negotiating a consensus between patient and provider understanding of the illness, goals, and treatment expectations and actively monitoring medication adherence. ${ }^{95}$

\section{Clinical Correlation: ESFT}

The ESFT model is designed to improve medication adherence through enhanced patient-clinician communication. ${ }^{96}$ ESFT takes into account 4 domains that influence patient satisfaction, adherence to recommended therapy, and health outcomes. It was initially developed for treating hypertension but can be applied to other illnesses. ${ }^{48}$ The strength of recommendation in favor of implementing ESFT is C (expert opinion). Four components should be explored with the patient:

$\mathbf{E}$ the patient's explanatory model of the illness

$\mathbf{S}$ social and financial barriers to adherence

$\mathbf{F}$ fears and concerns about the medication or its potential side effects

$\mathbf{T}$ therapeutic contracting and playback

The following questions can help elicit and guide the discussion of each component:

E What do you call your problem? What do you think caused it? Why do you think it started when it did? What does your sickness do to you? How does it work? How severe is it? Will it have a short or long course? What do you fear most about your sickness? What are the chief problems your sickness has caused for you? What kind of treatment do 
you think you should receive? What are the most important results you hope to receive from the treatment? ${ }^{97}$

$\mathbf{S}$ What is your health insurance situation? Do you have access to a pharmacy? Will you be able to afford the treatment? Would you prefer that I give you a less expensive medication that is equally effective for your problem but has more side effects?

F What are your concerns about the treatment? Are you afraid of potential side effects? How will the new treatment interact with other medications you are taking? Are you concerned that the medication will interfere with your life? Are you afraid of becoming addicted to the medication or "needing it to function"? Are there important people in your life who think you should not follow this treatment? Are you concerned others will think less of you or stop trusting you if they find out you are taking this medication? Is there something in your religious or spiritual life that goes against this treatment?

T Do you agree with the treatment we have discussed? Will you be able to carry it out? Could you repeat to me what we agreed you would do so that I know that I explained myself clearly? Is there anything else I can do to clarify the instructions about the treatment?

\section{Perspective}

It is unreasonable to think that family physicians alone can overcome mental health disparities faced by Latinos. Attention clearly must be paid to broader economic, sociocultural, and institutional barriers to care. However, specialists in family medicine have opportunities to play a key role in the recognition and management of depression in US Hispanics, because this minority group is more likely to seek treatment in primary care than in other clinical settings. Some promising clinical strategies include increasing the availability of professionally trained Spanish interpreters, assessing for health literacy barriers to communication, maintaining clinical vigilance for somatic expressions of psychological distress, assessing for culturally specific ways of expressing and understanding depression, respecting culturally mediated treatment preferences, actively negotiating patients' concerns and expectations regarding medication treatment, and providing case management services for patients at risk for nonadherence with antidepressant medications and counseling.

\section{References}

1. The Hispanic population in the United States: population characteristics: March 2002. Washington DC: US Bureau of the Census; 2003. Available from: http://www.census.gov/prod/2003pubs/p20-545.pdf

2. Fiscella K, Franks P, Doescher M, Saver B. Disparities in health care by race, ethnicity, and language among the insured: findings from a national sample. Med Care 2002;40:52-9.

3. Wells K, Klap R, Koike A, Sherbourne C. Ethnic disparities in unmet need for alcoholism, drug abuse, and mental health care. Am J Psychiatry 2001;158: 2027-32.

4. Young AS, Klap R, Sherbourne CD, Wells KB. The quality of care for depressive and anxiety disorders in the United States. Arch Gen Psychiatry 2001;58:5561.

5. Hixon A, Chapman R. Healthy People 2010: the role of family physicians in addressing health disparities. Am Fam Physician 2000;62:1971-2.

6. US Department of Health and Human Services. Mental health: culture, race, and ethnicity-a supplement to mental health: a report of the Surgeon General. Rockville (MD): Substance Abuse and Mental Health Services Administration, Center for Mental Health Services; 2001. Available from: http://www.surgeongeneral.gov/library/mentalhealth/cre/

7. Hough RL, Landsverk JA, Karno M, et al. Utilization of health and mental health services by Los Angeles Mexican Americans and non-Hispanic Whites. Arch Gen Psychiatry 1987;44:702-9.

8. Wells KB, Golding JM, Hough RL, Burnam MA, Karno M. Acculturation and the probability of use of health services by Mexican Americans. Health Serv Res 1989;24:237-57.

9. Vega WA, Kolody B, Aguilar-Gaxiola S, Catalano R. Gaps in service utilization by Mexican Americans with mental health problems. Am J Psychiatry 1999; 156:928-34.

10. Kemp BJ, Staples F, Lopez-Aqueres W. Epidemiology of depression and dysphoria in an elderly Hispanic population. J Am Geriatr Soc 1987;35:920-6.

11. Hoppe S, Leon R, Realini J. Depression and anxiety among Mexican Americans in a family health center. Soc Psychiatry Psychiatr Epidemiol 1989;24:63-8.

12. Miranda J, Azocar F, Komaromy M, Golding JM. Unmet mental health needs of women in publicsector gynecologic clinics. Am J Obstet Gynecol 1998;178:212-7.

13. Olfson M, Shea S, Feder A, et al. Prevalence of anxiety, depression, and substance use disorders in an urban general medicine practice. Arch Fam Med 2000;9:876-83.

14. Alegria M, Takeuchi D, Canino G, et al. Considering context, place and culture: the National Latino and Asian American Study. Int J Methods Psychiatr Res 2004;13:208-20 
15. Torre CA, Vecchini HR, Burgos W, eds. The commuter nation: perspectives on Puerto Rican migration. Rio Piedras (PR): University of Puerto Rico Press; 1994.

16. Miranda J, Hohmann AA, Attkisson CC. Epidemiology of mental disorders in primary care. In: Miranda J, Hohmann A, Attkisson CC, editors. Mental disorders in primary care. San Francisco: Jossey-Bass; 1994. p. 3-15.

17. Roberts R. Prevalence of depressive symptoms among Mexican Americans. J Nerv Ment Dis 1981; 169:213-9.

18. Robins L, Regier D. Psychiatric disorders in America: the epidemiologic catchment area study. New York: Free Press; 1991.

19. Kessler RC, McGonagle KA, Zhao S, et al. Lifetime and 12-month prevalence of DSM-III-R psychiatric disorders in the United States. Arch Gen Psychiatry 1994;51:8-19.

20. Kessler R, Berglund P, Demler O, et al. National Comorbidity Survey Replication. The epidemiology of major depressive disorder: results from the $\mathrm{Na}$ tional Comorbidity Survey Replication (NCS-R). JAMA 2003;289:3095-105.

21. Burnam M, Hough R, Escobar J, et al. Six-month prevalence of specific psychiatric disorders among Mexican Americans and non-Hispanic whites in Los Angeles. Arch Gen Psychiatry 1987;44:687-94.

22. Vega WA, Kolody B, Aguilar-Glaxiola S, Alderete E, Catalano R, Caraveo-Anduaga J. Lifetime prevalence of DSM-III-R psychiatric disorders among urban and rural Mexican Americans in California. Arch Gen Psychiatry 1998;55:771-8.

23. Demyttenaere K, Bruffaerts R, Posada-Villa J; WHO World Mental Health Survey Consortium. Prevalence, severity, and unmet need for treatment of mental disorders in the World Health Organization World Mental Health Surveys. JAMA 2004;291: 2581-90.

24. Narrow W, Rae D, Moscicki E, Locke B, Regier D. Depression among Cuban Americans: the Hispanic Health and Nutrition Examination Survey. Soc Psychiatry Psychiatr Epidemiol 1990;25:260-8.

25. Ortega A, Rosenheck R, Alegria M, Desai R. Acculturation and the lifetime risk of psychiatric and substance use disorders among Hispanics. J Nerv Ment Dis 2000;188:728-35.

26. Vera M, Alegria M, Freeman D, Robles R, Rios R, Rios C. Depressive symptoms among Puerto Ricans: island poor compared with residents of the New York City area. Am J Epidemiol 1991;134:502-10.

27. Hispanic health in the United States. Council on Scientific Affairs. JAMA 1991;265:248-52.

28. Oquendo M, Ellis S, Greenwald S, Malone K, Weissman M, Mann J. Ethnic and sex differences in suicide rates relative to major depression in the United States. Am J Psychiatry 2001;158:1652-8.

29. Rogler LH, Cortes DE, Malgady RG. Acculturation and mental health status among Hispanics. Convergence and new directions for research. Am Psychol 1991;46:585-97

30. Dixon LB, Sundquist J, Winkleby M. Differences in energy, nutrient, and food intakes in a US sample of Mexican-American women and men. Am J Epidemiol 2000;152:548-57.

31. Ring J, Marquis P. Depression in a Latino immigrant medical population. Am J Orthopsychiatry 1991;61:298-302.

32. Escobar J, Waitzkin H, Silver R, Gara M, Holman A. Abridged somatization: a study in primary care. Psychosom Med 1998;60:466-72.

33. Escobar J, Hoyos-Nervi C, Gara M. Immigration and mental health: Mexican Americans in the United States. Harv Rev Psychiatry 2000;8:64-72.

34. Spitzer RL, Kroenke K, Williams JBW. Validation and utility of a self-report version of PRIME-MD: the PHQ primary care study. JAMA 1999;282:173744.

35. Kroenke K, Spitzer R, Williams J. The PHQ-9: validity of a brief depression severity measure. J Gen Intern Med 2001;16:606-13.

36. US Bureau of the Census. The Hispanic population of the United States. Washington DC: US Government Printing Office; 2000. Available from: http://www.census.gov/population/www/socdemo/ hispanic.html

37. Perez-Stable E, Napoles-Springer A, Miramontes J. The effects of ethnicity and language on medical outcomes of patients with hypertension or diabetes. Med Care 1997;12:1212-9.

38. Morales LS, Cunningham WE, Brown JA, Liu H, Hays RD. Are Latinos less satisfied with communication by health care providers? J Gen Intern Med 1999;14:409-17.

39. Williams M, Baker D, Parker R, Nurss J. Relationship of functional health literacy to patients' knowledge of their chronic disease. Arch Intern Med 1998; 158:166-72.

40. Parker R, Jacobson T. The role of health literacy in narrowing the treatment gap for hypercholesterolemia. Am J Managed Care 2000;6:1340-2.

41. Schillinger D, Grumbach K, Piette J, et al. Association of health literacy with diabetes outcomes. JAMA 2002;288:475-82.

42. Ruiz P. Hispanic access to health/mental health services. Psychiatr Q 2002;73:85-91.

43. Woloshin S, Bickell NA, Schwartz LM, Gany F, Welch HG. Language barriers in medicine in the United States. JAMA 1995;273:724-8.

44. Hornberger J, Itkakura H, Wilson S. Bridging language and cultural barriers between physicians and patients. Public Health Rep 1997;112:410-7.

45. Jacobs E, Lauderdale D, Meltzer D, Shorey J, Levinson $W$, Thisted R. Impact of interpreter services on delivery of health care to limited English-proficiency patients. J Gen Intern Med 2001;16:468-74. 
46. Cultural Competence standards in managed care mental health services: four underserved/ underrepresented racial/ethnic groups [monograph on the Internet]. Washington DC: Center for Mental Health Services, Substance Abuse and Mental Health Services Administration, US Department of Health and Human Services; 2001 [cited 2004 Jan 12]. Available from: http://www.mentalhealth. samhsa.gov/publications/allpubs/SMA00-3457/ default.asp

47. Federal Interagency Working Group on Limited English Proficiency [homepage on the Internet]. Washington DC: US Department of Justice; c2002 [ updated 2002 Oct 31; cited 2004 Dec 22]. Know your rights: limited English proficiency; [about 3 screens]. Available from: http://www.lep.gov/ benebroch.htm. American Medical Association [homepage on the Internet]. Chicago: The Association; c1995-2002 [updated 2001 Aug 23; cited 2002 Aug 12]. AMA Office of Group Practice Liaison; [about 2 screens]. Available from: http://www.amaassn.org/ama/pub/category/1736.html

48. Lewis-Fernández R, Like R, Guarnaccia PJ, Betancourt J. Building cultural competency in clinical practice: a workshop to increase the proficiency of physicians, nurses, and staff who provide care to diverse Hispanic/Latino patients/clients. A CME workshop accredited by the University of Medicine and Dentistry of New Jersey, Santa Barbara, CA 2004.

49. Cornelio M. Legal issues in the translation of healthcare documents. Am Translators Assoc Chronicle 2002;31:24-8.

50. Kleinman AM, Good BJ, editors. Culture and depression: studies in the anthropology and cross-cultural psychiatry of affect and disorder. Berkeley: University of California Press; 1995.

51. Kirmayer LJ, Young A. Culture and somatization: clinical, epidemiological, and ethnographic perspectives. Psychosom Med 1998;60:420-30.

52. Mezzich J, Raab E. Depressive symptomatology across the Americas. Arch Gen Psychiatry 1980;37: $818-23$.

53. Escobar J, Gomez J, Tuason V. Depressive symptomatology in North and South American patients. Am J Psychiatry 1983;140:47-51.

54. Kolody B, Vega W, Meinhardt K, Bensussen G. The correspondence of health complaints and depressive symptoms among Anglos and Mexican-Americans. J Nerv Ment Dis 1986;174:221-28.

55. Canino GJ, Bird HR, Shrout PE, et al. The prevalence of specific psychiatric disorders in Puerto Rico. Arch Gen Psychiatry 1987;44:727-35.

56. Canino IA, Rubio-Stipec M, Canino G, Escobar JI. Functional somatic symptoms: a cross-ethnic comparison. Am J Orthopsychiatry 1992;62:605-12.

57. Escobar J, Burnham M, Karno M, Forsythe A, Gold- ing J. Somatization in the community. Arch Gen Psychiatry 1987;44:713-8.

58. Escobar JI, Canino G. Unexplained physical complaints. Psychopathology and epidemiological correlates. Br J Psychiatry Suppl 1989;(4):24-7

59. Guarnaccia P, Angel R, Worobey J. The factor structure of the CES-D in the Hispanic Health and Nutrition Examination Survey. Soc Sci Med 1989; 29:85-94.

60. Rosen G, Kleinman A, Katon W. Somatization in family practice: a biopsychosocial approach. J Fam Practice 1982;14:493-502.

61. Harwood A. Ethnicity and medical care. Cambridge (MA): Harvard University Press; 1981.

62. Guarnaccia P, Lewis-Fernández R, Rivera Marano M. Toward a Puerto Rican popular nosology: Nervios and ataques de nervios. Cult Med Psych 2003;27: 339-66.

63. Lewis-Fernández R, Guarnaccia PJ, Patel S, Lizardi D, Diaz N. Ataque de nervios: anthropological, epidemiological, and clinical dimensions of a cultural syndrome. In Georgiopoulos AM, Rosenbaum JF, editors. Perspectives in cross-cultural psychiatry. Philadelphia: Lippincott Williams \& Wilkins; 2005. p. $63-85$.

64. Nichter M. Idioms of distress: alternatives in the expression of psychosocial distress. Cult Med Psych 1981;5:379-408.

65. Guarnaccia PJ, Canino G, Rubio-Stipec M, Bravo M. The prevalence of ataques de nervios in the Puerto Rico Disaster Study. J Nerv Ment Dis 1993; 181:157-65.

66. Lewis-Fernández R. Assessing psychosis screeners among underserved urban primary care patients. $\mathrm{Pa}$ per presented at 15th Annual Scientific Symposium of the National Alliance for Research on Schizophrenia and Depression; 2003 Oct 17-18; New York, New York. Great Neck (NY): National Alliance for Research on Schizophrenia and Depression; 2003.

67. Lu FG, Lim RF, Mezzich JE. Issues in the assessment and diagnosis of culturally diverse individuals. In: Oldham JM, Riba MB, editors. American Psychiatric Press Review of Psychiatry. Vol. 14. Washington DC: American Psychiatric Press; 1995. p. $477-$ 510.

68. Lewis-Fernández R, Díaz N. The cultural formulation: a method for assessing cultural factors affecting the clinical encounter. Psychiatr Q 2002;73:271-95.

69. American Psychiatric Association. Diagnostic and statistical manual of mental disorders, 4th ed (DSMIV). Washington DC: APA Press; 1994.

70. Dwight-Johnson M, Sherbourne CD, Liao D, Wells KB. Treatment preferences among depressed primary care patients. J Gen Intern Med 2000;15:52734.

71. Cooper LA, Gonzales JJ, Gallo JJ, et al. The acceptability of treatment for depression among African- 
American, Hispanic, and White primary care patients. Med Care 2003;41:479-89.

72. Dwight-Johnson M, Lagomasino IT, Aisenberg E, Hay J. Using conjoint analysis to assess depression treatment preferences among low-income Latinos. Psychiatr Serv 2004;5 5:934-6.

73. Miranda J, Azocar F, Organista K, Dwyer E, Arean P. Treatment of depression among impoverished primary care patients from ethnic minority groups. Psychiatr Serv 2003;54:219-25.

74. Rosselló J, Bernal G. The efficacy of cognitive-behavioral and interpersonal treatments for depression in Puerto Rican adolescents. J Consult Clin Psychol 1999;67:734-45.

75. Escobar JI, Tuason VB. Antidepressant agents: a cross cultural study. Psychopharm Bull 1980;16:4952.

76. Lin K-M. Biological differences in depression and anxiety across races and ethnic groups. J Clin Psychiatry 2001;62 Suppl 13:13-9; discussion 20-1.

77. Marin H, Escobar JI. Special issues in the psychopharmacological management of US Hispanics. Psychopharmacol Bull 2001;35:197-212.

78. Sánchez-Lacay JA, Lewis-Fernández R, Goetz D, et al. Open trial of nefazodone among Hispanics with major depression: efficacy, tolerability, and adherence issues. Depress Anxiety 2001;13:118-24.

79. Plewes JM, Bailey RK, Mallinckrodt CH, et al. Duloxetine for the treatment of major depressive disorder in Hispanic and African American patients. Paper presented at American Psychiatric Association Annual Meeting; 2004 May 1-6; New York, New York. Arlington (VA): American Psychiatric Association; 2004.

80. Skaer TL, Sclar DA, Robison LM, Galin RS. Trends in the rate of depressive illness and use of antidepressant pharmacotherapy by ethnicity/race. Clin Ther 2000;22:1575-89.

81. Sleath B, Rubin R, Huston S. Antidepressant prescribing to Hispanic and non-Hispanic white patients in primary care. Ann Pharmacother 2001;35: 419-23.

82. Borowsky SJ, Rubenstein LV, Meredith LS, Camp $\mathrm{P}$, Jackson-Triche M, Wells KB. Who is at risk of nondetection of mental health problems in primary care? J Gen Intern Med 2000;15:381-88.

83. Sclar DA, Robinson LM, Skaer TL, Galin RS. Ethnicity and the prescribing of antidepressant pharmacotherapy: 1992-1995. Harv Rev Psychiatry 1999;7: 29-36.

84. Miranda J, Duan N, Shebourne C, et al. Improving care for minorities: can quality improvement inter- ventions improve care and outcomes for depressed minorities? Health Serv Res 2003;38:613-30.

85. Sirey JA, Meyers BS, Bruce ML, Alexopoulos GS, Perlick DA, Raue P. Predictors of antidepressant prescription and early use among depressed outpatients. Am J Psychiatry 1999;156:690-6.

86. Harman JS, Edlund MJ, Fortney JC. Disparities in the adequacy of depression treatment in the United States. Psychiatr Serv 2004;55:1379-85.

87. Alonso M, Val E, Rapaport MH. An open-label study of SSRI treatment in depressed Hispanic and non-Hispanic women [letter]. J Clin Psychiatry 1997;58:31

88. Wagner GJ, Maguen S, Rabkin JG. Ethnic differences in response to fluoxetine in a controlled trial with depressed HIV-positive patients. Psychiatr Serv 1998;49:239-40.

89. Marcos LR, Cancro R. Pharmacotherapy of Hispanic depressed patients: clinical observations. Am J Psychotherapy 1982;36:505-12.

90. Sleath B, Rubin RH, Huston SA. Hispanic ethnicity, physician-patient communication, and antidepressant adherence. Compr Psychiatry 2003;44: 198-204.

91. Lawson H, Kahn M, Heiman E. Psychopathology, treatment outcome and attitude toward mental illness in Mexican American and European patients. Int J Soc Psychiatry 1982;28:20-6.

92. Pol LG, Mueller KJ, Adidam PT. Racial and ethnic differences in health insurance for the near elderly. J Health Care Poor Underserved 2003;23:229-40.

93. Gary TL, Narayan KM, Gregg EW, Beckles GL, Saaddine JB. For the patient. Racial/ethnic differences in the health care experience of US adults with diabetes. Ethn Dis 2003;13:154.

94. Economic Research Initiative on the Uninsured [homepage on the Internet]. Ann Arbor (MI): University of Michigan; c2001 [updated 2001; cited 13 Jan 2005]. Available from: http://www.umich.edu/ $\sim$ eriu/fastfacts/meps2001_4_pit.html

95. Bull S, Hu X, Hunkeler E, et al. Discontinuation of use and switching of antidepressants: influence of patient-physician communication. JAMA 2002;288: 1403-9.

96. Betancourt J, Carrillo JE, Green AR. Hypertension in multicultural and minority populations: linking communication to compliance. Curr Hypertens Rep 1999;1:482-8.

97. Kleinman A. Patients and healers in the context of culture. Berkeley: University of California Press; 1980. 